\title{
PHOTOIONIZATION FROM EXCITED STATES OF HELIUM
}

\section{L. JACOBS}
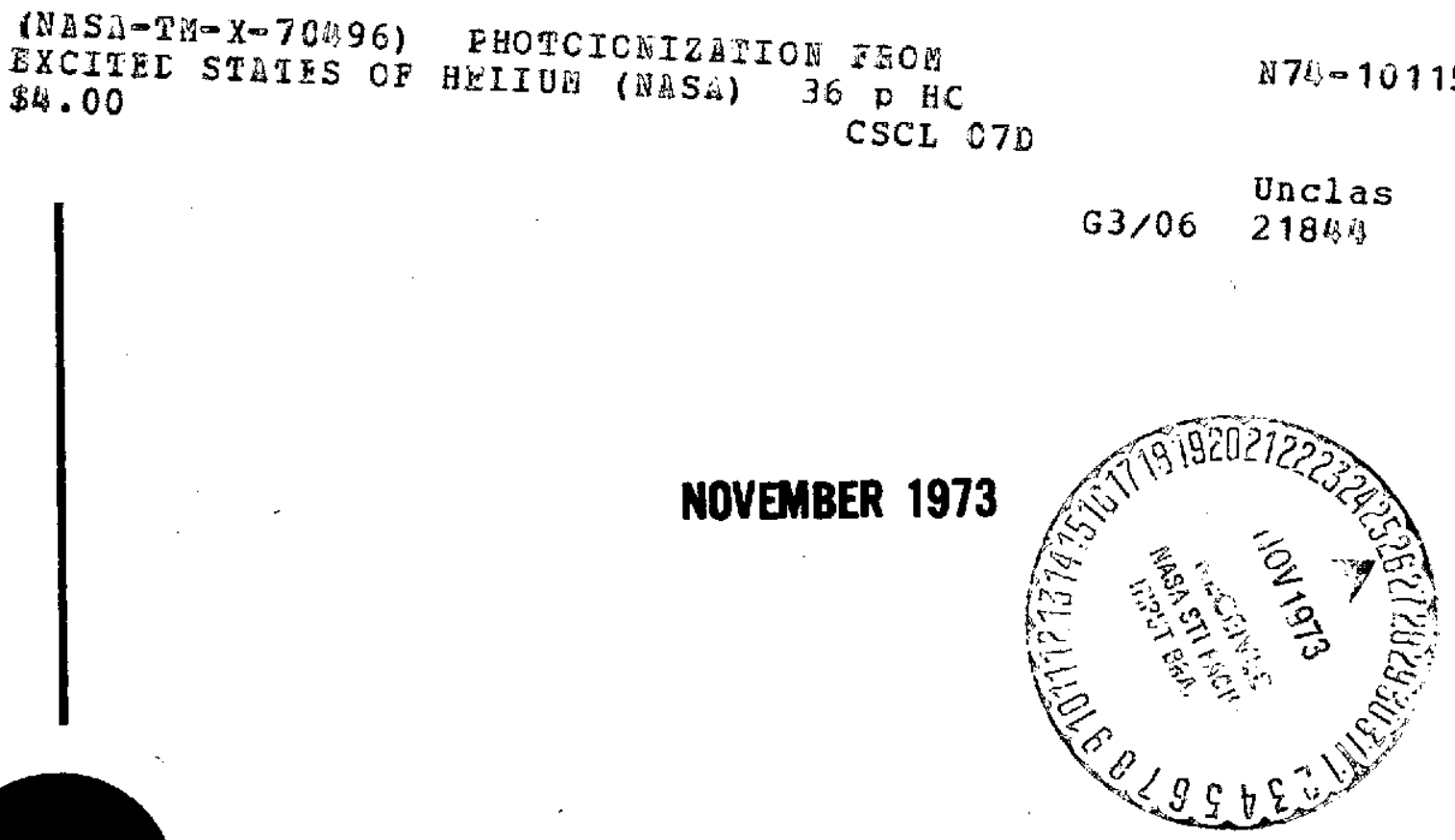
PHOTOIONIZATION FROM EXCITED STATES OF HELIUM

V. L. JACOBS*

THEORETICAL STUDIES BRANCH

LABORATORY FOR SPACE PHYSICS

GODDARD SPACE FLIGHT CENTER

NATIONAL AERONAUTICS AND SPACE ADMINISTRATION

GREENBELT, MARYLAND 20771

* National Academy of Sciences-National Research Council Resident Research Associate 


\section{ABSTRACT}

The cross sections for phototonfzation from the $2^{I} S, 2^{3} \mathrm{~S}, 2^{1} \mathrm{P}$ and ${ }^{3} \mathrm{P}$ excited states of helium are calculated for photoelectron energies below the $n=2$ threshold of $\mathrm{He}^{+}$using Hylleraas bound state wave functions and $1 s-2 s-2 p$ close coupling final state wave functions. The resonant structures associated with the lowest-1ying ${ }^{1},{ }_{\mathrm{P}},{ }_{\mathrm{P}}$, and ${ }_{\mathrm{D}}$ autoionizing states of helium are found to be characterized by large values of the line profile parameter q. The cross sections and the photoelectron angular distribution asymmetry parameters for the P-states are calculated for various polarization states of the target atom and the incident photon. Experiments which would lead to the separate determinations of the S- and D- wave partial photoionization cross sections are discussed. 


\section{Introduction}

Photoionization from excited states of helium plays an important role in the transfer of radiation through laboratory plasmas ${ }^{1}$ and hot stellar atmospheres ${ }^{2}$. In addition, accurate cross sections for singleand multi-photon ionization from excited states of helium may be required for a quantitative understanding of the interaction of laser radiation with a helium discharge. ${ }^{3}$ Percent advances in atomic beam methods and in frequency-tunable laser techniques have led to an accurate experimental determination of the photoionization cross sections for the $2^{1} \mathrm{~S}$ and $2^{3} \mathrm{~S}$ metastable states of helium in the near-threshold region. ${ }^{4}$

Accordingly, the theoretical investigation of photoionization processes from excited states of helium is worthwhile.

In this paper, the cross sections for single-photon ionization from the $2^{1} \mathrm{~S}, 2^{3} \mathrm{~S}, 2^{1} \mathrm{P}$, and $2^{3} \mathrm{P}$ states of helium are evaluated for photoelectron energies below the $n=2$ threshold of the residual $\mathrm{He}^{+}$ion, using $1 s-2 s-2 p$ close coupling final state continuum wave functions. Results for the $2^{1} \mathrm{~S}$ cross section in the nonresonant region have been reported in two earlier papers ${ }^{5,6}$ and the near-threshold values ${ }^{6}$ are in good agreement with the experimental determination. More recent theoretical studies of photoionization from the $1^{1} S, 2^{1} S$, and $2^{3} s$ states of helium were restricted to photoelectron energies above the $n=2$ threshold ${ }^{7-9}$ where transitions can occur to the $2 \mathrm{~s}$ and $2 \mathrm{p}$ excited states of $\mathrm{He}^{+}$and the resulting photoelectron angular distribution is a function of energy. From these studies and from the results 
of a recent helium photoionization experiment, 10 it has become apparent that the inclusion of close coupling between degenerate excited states of the $\mathrm{He}^{+}$ion is essential not only for the accurate calculation of the resonance properties below the excitation threshold, ${ }^{11}$ but also for the correct determination of the distribution between the degenerate lonic states above the threshold.

Norcross ${ }^{12}$ has evaluated the $2^{1} \mathrm{~S}$ and $2^{3} \mathrm{~S}$ helfum photoionization cross sections using $1 s-2 s-2 p$ close coupling continum wave functions, but he employed Hartreeflock bound state wave functions which are not expected to be as accurate as the Hylleraas wave functions used in the present calculation. In previous evaluations of the $2^{1} P$ and $2^{3} P$ helium photolonization cross sections, the final continuum states were represented by hydrogenic ${ }^{13}$ and quantum defect ${ }^{14,15}$ wave functions. These approximations may provide reliable estimates in the near-threshold region, but they cannot account for the prominent resonant features which are predicted by the $1 \mathrm{~s}-2 \mathrm{~s}-2 \mathrm{p}$ close coupling calculations.

An important feature of photoionization from exclted states of helium is the significant enhancement of the total cross sections which results from taking into account transitions to excited states of the $\mathrm{He}^{+}$ion. It is therefore not surprising that the resonant structure associated with the $n=2$ threshold has been found to be more conspicuous in the photoionization continua of the metastable states than in the ground state continuum. This fact is clearly illustrated by the line profile parameters reported by Norcross ${ }^{12}$ for the lowest $\operatorname{six}^{1} \mathbf{P}$ resonance in the $2^{1} \mathrm{~S}$ cross section which are in some cases more than a hundred times 
larger than the corresponding parameters obtained for the helium ground state by Burke and McVicar. 11

The study of photoionization processes from the $2^{1} \mathrm{P}$ and $2^{3} \mathrm{P}$ states of helium would be difficult with present experimental techniques because of the short radiative ilfetimes of these excited states in comparison with the helium metastable states. However, photoabsorption from the P- states using laser light may ultimately provide the most precise method for the experimental investigation of the S- and D- wave ionization continua of the helium atom.

In contrast to photoionization from the helium S- states, photoelectric transitions from the $\mathrm{P}$ - states will in: general give rise to an energy-dependent photoelectron angular distribution below the $\mathrm{n}=2$ threshold. In the case of unpolarized P- states the angular distribution can be characterized by a single asymmetry parameter $\beta$ regardless of the polarization state of the incident radiation. ${ }^{16-19}$ The angular distribution of photoelectrons from polarized P- states can be determined from a knowledge of two asymmetry parameters, which may show a complicated dependence on the initial polarization states.

An important simplification would occur if the helium atom could first be excited into an aligned $P-$ state and then ionized by linearly or circularly polarized photons incident in a suitable direction with respect to the atomic quantization axis. In these cases, the D- partial wave ionization continum will be observed directly, and the photoelectron angular distribution below the $n=2$ threshold will not depend on the incident photon energy. 


\section{The Theory of Differential Photoionization Cross Sections}

A formulation of the theory of differential cross sections for atomic photoelectric transitions has been developed in terms of the irreducible tensor components of the initial and final polarization states. 19 In this section it is appropriate to present the results of this formulation which are applicable when the ejected electron and the residual ion polarizations are not measured. The discussion will be further restricted to cases where the coordinate system can be chosen to reduce the form of the differential cross section (for the transition to the $n l_{1}$ state of $\mathrm{He}^{+}$) to the Legendre polynomial expansion

$$
\sigma_{\mathrm{n} \ell_{1}}\left(\overrightarrow{\mathrm{k}}_{\mathrm{n}}\right)=\frac{\sigma_{\mathrm{n} \ell_{1}}}{4 \pi}\left[1+\sum_{\mathrm{L}>0} \beta \mathrm{n} \ell_{1}, \mathrm{~L} \mathrm{P}_{\mathrm{L}}(\cos \theta)\right]
$$

where the approprlate definition of $\theta$ will depend on the initial polarization states. It is important to note that the electric-dipole selection rules allow only a few even values of $L$ to contribute.

The initial bound state of the helium atom may be specified by a principle quantum number $\mathrm{n}_{0}$ and by the total orbital and spin angular momenta $\mathrm{L}_{o}$ and $\mathrm{S}_{\mathrm{O}}$. The polarization state of the helium atom with respect to the orbital angular momentum $L_{0}$ may be represented by a $\left(2 \mathrm{~L}_{0}+1\right) \mathrm{x}$ (2 $\mathrm{L}_{\mathrm{o}}+1$ j-density matrix $\rho_{\mathrm{o}}$, which can be expressed in terms of the complete set of $\left(2 \mathrm{~L}_{\mathrm{o}}+1\right)^{2}$ irreducible tensor operators ${ }^{20}$ in the form of the multipole expansion

$$
\left\langle\begin{array}{lll}
\mathrm{ML}_{\mathrm{o}} & \left|\rho_{\mathrm{o}}\right| & \mathrm{ML}_{\mathrm{o}}^{1}
\end{array}\right\rangle=\sum_{\mathrm{K}_{\mathrm{o}} \mathrm{N}_{\mathrm{o}}} \rho_{\mathrm{o}}\left(\mathrm{K}_{\mathrm{o}}, \mathrm{N}_{\mathrm{o}}\right)(-1)^{\mathrm{L}_{\mathrm{o}}-\mathrm{ML}_{0} \sqrt{\left(2 \mathrm{~K}_{\mathrm{o}}+1\right)}}\left(\begin{array}{c}
\mathrm{L}_{\mathrm{o}} \mathrm{K}_{\mathrm{o}} \mathrm{L}_{\mathrm{o}} \\
-\mathrm{ML}_{\mathrm{o}} \mathrm{N}_{\mathrm{o}} \mathrm{ML}_{\mathrm{o}}^{1}
\end{array}\right)
$$


Similarly, the : Jlarization state of the incident electric-dipole photon may be represented by a $3 \times 3$ density utatrix $\rho_{\mathrm{I}}$, which will depend on the orientation of the photon beam with respect to the atomic quantization axis. It will also be convenient to expand $\rho_{\mathrm{r}}$ in the form (2) with irreducible tensor components denoted by $\rho_{\mathrm{r}}\left(\mathrm{K}_{\mathrm{r}}, \mathrm{N}_{\mathrm{r}}\right)$. Explicit expressions for the irreducible tensor components of the initial polarization states which are treated in this paper have been given previously. 19

The final state continuum channels of the $\mathrm{He}^{+}-\mathrm{e}^{-}$system are defined by the quantum numbers $n \ell_{1} \ell_{2}, \mathrm{~L}_{\mathrm{T}} \mathrm{S}_{\mathrm{O}}$, where $\ell_{2}$ is the photoelectron relative orbital angular momentum and $\mathrm{L}_{\mathrm{T}}$ is the total orbital angular momentum.

The expressions which are derived for the total cross section $\sigma_{\mathrm{n} \ell_{1}}$ (in units of $\mathrm{a}_{0}^{2}$ ) and the asymmetry parameters $\beta \mathrm{n} l_{1}, \mathrm{~L}$ may be written in the forms

$$
\begin{gathered}
\sigma_{n \ell_{1}}=4 \pi^{2} \alpha \omega \sum_{\ell_{2} L_{T}} \sum_{K}(-1)^{1+L_{o}+L_{T}}\left\{\begin{array}{ccc}
L_{o} & L_{o} & K \\
1 & 1 & L_{T}
\end{array}\right\} \\
\times \rho_{o}(k, o) \rho_{r}(k, o)\left|M\left(\ell_{2}, L_{T}\right)\right|^{2}
\end{gathered}
$$

and 


$$
\begin{aligned}
& \beta \mathrm{n} \ell_{1}, \mathrm{~L}=\frac{4 \pi^{2} \alpha \omega}{\sigma_{\mathrm{n} \ell_{1}}} \sum_{\ell_{2}, \mathrm{~L}_{\mathrm{T}}} \sum_{\ell_{2}^{\prime}, \mathrm{L}_{\mathrm{T}}} \sum_{\mathrm{K}_{\mathrm{o}}} \sum_{\mathrm{K}_{\mathrm{I}}}(-1)^{\ell_{1}+\mathrm{L}_{\mathrm{T}}} \\
& X(2 \mathrm{~L}+1) \sqrt{\left(2 \mathrm{~K}_{\mathrm{o}}+1\right)\left(2 \mathrm{~K}_{\mathrm{r}}+1\right)\left(2 \mathrm{\ell}_{2}+1\right)\left(2 \ell_{2}^{\prime}+1\right)\left(2 \mathrm{~L}_{\mathrm{T}}+1\right)\left(2 \mathrm{~L}_{\mathrm{T}}^{\prime}+1\right)} \\
& \times\left(\begin{array}{ccc}
\ell_{2} & l_{2}^{\prime} & \mathrm{L} \\
0 & 0 & \mathrm{a}
\end{array}\right)\left(\begin{array}{ccc}
\mathrm{K}_{\mathrm{o}} & \mathrm{K}_{\mathrm{r}} & \mathrm{L} \\
0 & 0 & 0
\end{array}\right)\left\{\begin{array}{lll}
\ell_{2} & \ell_{2}^{\prime} & \mathrm{L} \\
\mathrm{L}_{\mathrm{T}}^{\prime} & \mathrm{L}_{\mathrm{T}} & \ell_{1}
\end{array}\right\}\left\{\begin{array}{ccc}
\mathrm{L}_{\mathrm{o}} & 1 & \mathrm{~L}_{\mathrm{T}}^{\prime} \\
\mathrm{L}_{\mathrm{o}} & 1 & \mathrm{~L}_{\mathrm{T}} \\
\mathrm{K}_{\mathrm{o}} & \mathrm{K}_{\mathrm{r}} & \mathrm{L}
\end{array}\right\} \\
& X \rho_{\mathrm{o}}\left(\mathrm{K}_{\mathrm{o}}, \mathrm{o}\right) \rho_{\mathrm{r}}\left(\mathrm{K}_{\mathrm{r}}, \mathrm{o}\right) \mathrm{M}\left(\ell_{2}, \mathrm{~L}_{\mathrm{T}}\right) \mathrm{M}\left(\ell_{2}^{\prime}, \mathrm{L}_{\mathrm{T}}^{\prime}\right)^{*}
\end{aligned}
$$

where $\omega$ is the incident photon energy measured in Rydbergs $\left(\omega=I_{0}+k_{1}^{2}\right.$, where $I_{0}$ is the ionization potential), and $\alpha$ is the fine structure constant. The summations are to be taken over all values of the angular momenta which are consistent with the selection rules associated with the $3-j, 6-j$, and $9-j$ symbols. The transition amplitudes $M\left(l_{2}, L_{T}\right)$ may be defined in terms of either the dipole length or the dipole velocity forms of the matrix elements as follows

$$
\mathrm{M}^{(\mathrm{L})}\left(\ell_{2}, \mathrm{~L}_{\mathrm{T}}\right)=\mathrm{e}^{\mathrm{i}\left(\sigma_{\ell_{2}}-\ell_{2} \pi / 2\right)}\left(\mathrm{n} \ell_{1} \ell_{2}, \mathrm{~L}_{\mathrm{T}} \mathrm{S}_{\mathrm{o}}\left\|\overrightarrow{\mathrm{r}}_{1}+\overrightarrow{\mathrm{r}}_{2}\right\| \mathrm{n}_{\mathrm{o}} \mathrm{L}_{\mathrm{o}} \mathrm{S}_{\mathrm{o}}\right)
$$

$$
M^{(V)}\left(\ell_{2}, L_{T}\right)=\frac{2 \mathrm{e}^{\mathrm{i}\left(\sigma_{\ell_{2}}-\ell_{2} \pi / 2\right)}}{\omega}\left(\mathrm{n}_{1} \ell_{2}, \mathrm{~L}_{\mathrm{T}} \mathrm{S}_{\mathrm{o}}\left\|\vec{\nabla}_{1}+\vec{\nabla}_{2}\right\| \mathrm{n}_{\mathrm{o}} \mathrm{L}_{\mathrm{o}} \mathrm{S}_{\mathrm{o}}\right)
$$


where $\sigma_{\ell_{2}}$ denotes the Coulomb phase shift for the $\mathrm{He}^{+}$ion. In the reduced matrix elements of the dipole operators the final continuum states are assumed to be normalized to a Dirac delta function in the photoelectron energy.

The theory presented up to this point can be applied to any helium transition and, in fact, to any atomic system for which the LS representation is appropriate. In the remainder of this paper only transitions to $\mathrm{He}^{+}(\mathrm{ls})$ will be treated, and therefore the subscripts $\mathrm{n}_{1}$ will be omitted.

The photoelectron angular distribution resulting from ionization of unpolarized helium atoms is given by equation (1) with only the $\mathrm{L}=2$ term included in the summation. The associated asymetry parameter is customarily denoted by $\beta$. When the incident radiation is linearly polarized $\theta$ measures the angle between the photoelectron momentum $\overrightarrow{\mathrm{k}}_{1}$ and the photon polarization direction. For unpolarized or circularly polarized photons it is necessary only to replace $\beta$ by $-\beta / 2$ and to define $\theta$ as the angle between the photoelectron and incident photon propagation directions. For photolonization from the helium S- states, the well-known result that $\beta$ is identically 2 , independent of energy, can be recovered from equations (3) and (4). In the case of unpolarized P-states, the interference between the transition amplitudes corresponding to the S- and D- channels will result in an energy-dependence of the asymmetry parameter $\beta$ which is expected to be rapid in the vicinity of an autoionization feature. 
The photoelectron angular distribution from aligned ( $\mathrm{ML}_{0}=1$ )

P-states of helium is determined by a knowledge of two asymmetry parameters, corresponding to $\mathrm{L}=2$ and 4 . In the cases of incident photons with linear polarization in the direction of the atomic quantization axis and unpolarized or right circularly polartzed photons propagating along the atomic quantization axis, the definitions given previously for $\theta$ still apply. For the circular and linear polarfzations, only the D-partial wave channel will be exclted in the photoelectric transition and the asymmetry parameters $\beta_{2}$ and $\beta_{4}$ w111 then be independent of energy. 
III. The Helium Wave Functions

The $2^{1} \mathrm{~S}, 2^{3} \mathrm{~S}, 2^{1} \mathrm{P}$, and $2^{3} \mathrm{P}$ bound excited states of the helium atom are represented with high precision by the Hylleraas wave functions

$$
\begin{aligned}
\Psi_{\mathrm{n}_{\mathrm{o}} \mathrm{L}_{\mathrm{o}} \mathrm{s}_{\mathrm{o}}}\left(\overrightarrow{\mathrm{r}}_{1}, \overrightarrow{\mathrm{r}}_{2}\right) & =\left[\frac{1+(-1)^{\mathrm{s}_{\mathrm{o}}} \mathrm{p}_{12}}{\sqrt{2}}\right] \mathrm{e}^{-\mathrm{ar}_{1}-\mathrm{br}_{2}} \\
& \times \sum_{\mathrm{ijk}} \mathrm{C}_{\mathrm{ijk}} \mathrm{r}_{1}^{\mathrm{i}} \mathrm{r}_{2}^{\mathrm{j}} \mathrm{r}_{12}^{\mathrm{k}} \operatorname{Yoo}\left(\hat{\mathrm{r}}_{1}\right) \mathrm{YL}_{\mathrm{o}} \mathrm{ML}_{\mathrm{o}}\left(\hat{\mathrm{r}}_{2}\right)
\end{aligned}
$$

where $P_{12}$ is the electron exchange operator. The values for the exponential parameters $a$ and $b$ were taken from the work of Pekeris and co-workers ${ }^{21}$, and 56-term expansions were computed by diagonalizing the matrices of the Hamiltonian operator.

The ${ }^{1_{L_{T}}}$ and ${ }^{3} L_{\mathrm{T}}$ final state continuum wave functions with $\mathrm{L}_{\mathrm{T}}=0,1,2$ are expanded in the close coupling form ${ }^{22}$

$$
\begin{aligned}
& \Psi_{1 \mathrm{~s}, \ell_{2}}^{(-)}=\mathrm{L}_{\mathrm{T}}, \mathrm{S}\left(\overrightarrow{\mathrm{r}}_{1}, \overrightarrow{\mathrm{r}}_{2}\right)=\left[\frac{1+(-1)^{\mathrm{S}} \mathrm{P}_{12}}{\sqrt{2}}\right] \sum_{\mathrm{n}^{\prime} \ell_{1}^{\prime} \ell_{2}^{\prime}} \frac{\mathrm{Pn}^{\prime} \ell_{1}^{\prime}\left(\mathrm{r}_{1}\right)}{\mathrm{r}_{1}} \frac{\mathrm{Fn}^{\prime} \ell_{1}^{\prime} \ell_{2}\left(\mathrm{r}_{2}\right)}{\mathrm{r}_{2}} \\
& \times \sum_{\mathrm{m}_{1}^{\prime} \mathrm{m}_{2}^{\prime}}\left\langle\ell_{1}^{\prime} \ell_{2}^{\prime}, \mathrm{L}_{\mathrm{T}} \mathrm{ML}_{\mathrm{T}} \mid \ell_{1}^{\prime} \mathrm{m}_{1}^{\prime}, \ell_{2}^{\prime} \mathrm{m}_{2}^{\prime}\right\rangle \mathrm{Y}_{\ell_{1}^{\prime}\left(\hat{\mathrm{r}}_{1}\right)}^{\mathrm{m}_{1}^{\prime}} \mathrm{Y}_{\ell_{2}^{\prime}\left(\hat{\mathrm{r}}_{2}\right)}^{\mathrm{m}_{2}^{\prime}}
\end{aligned}
$$

where $\mathrm{Pn}^{\prime} \ell_{1}^{\prime}\left(\mathrm{r}_{1}\right)$ is the reduced $\mathrm{He}^{+}$ion radial wave function. The incoming wave boundary condition appropriate to the description of the photoionization process is indicated by the superscript (-). 
The coupled integro-differential equations which are satisfied by the ejected electron radial wave functions $F_{n^{\prime} \ell_{1} \ell_{2}^{\prime}}^{(-)}\left(r_{2}\right)$ were solved in the $1 s-2 s-2 p$ close-coupling approximation for the photoelectron energy range $0.15 \leqslant k_{1}{ }^{2} \leqslant 2.80$ using the collision code of $P$. G. Burke. ${ }^{11}$ The photoelectric transition matrix elements were then evaluated from the numerical continuum wavefunctions and the bound state weight functions as described previous $1 y .5,11$ 
IV The Photoionization Cross Sections

The $\mathrm{L}_{\mathrm{T}}$ - wave partial cross section for the photoelectric transition

$$
\mathrm{He}\left(\mathrm{n}_{\mathrm{o}} \mathrm{L}_{\mathrm{o}} \mathrm{S}_{\mathrm{o}}\right)+\mathrm{X} \omega \rightarrow \mathrm{He}^{+}(1 \mathrm{~s})+\mathrm{e}^{-}\left(\ell_{2}=\mathrm{L}_{\mathrm{T}}\right)
$$

from an unpolarized $L_{o}$ - state of helium, is conveniently expressed in terms of the osct1lator strength per unit Rydberg energy interval $\mathrm{df}_{\mathrm{L}_{\mathrm{T}}} / \mathrm{dE}$ according to the relation

$$
\sigma_{\mathrm{L}_{\mathrm{T}}}=4 \pi^{2} \alpha \frac{\mathrm{df}_{\mathrm{L}_{\mathbf{T}}}}{\mathrm{dE}}
$$

The ascillator strength may be defined in terms of the transition amplitudes introduced in the first section by

$$
\frac{\mathrm{df}_{\mathrm{L}_{\mathrm{T}}}}{\mathrm{dE}}=\frac{\omega}{3\left(2 \mathrm{~L}_{\mathrm{o}}+1\right)}\left|\mathrm{M}\left(\ell_{2}=\mathrm{L}_{\mathrm{T}}, \mathrm{L}_{\mathrm{T}}\right)\right|^{2}
$$

It is important to note that the cross section $\sigma_{\mathbf{L}_{\mathrm{T}}}$ given by equation (i3) is independent of the photon polarization.

The oscillator strengths assoctated with the $2^{1} \mathrm{~S} \rightarrow \mathrm{E}^{1} \mathrm{P}$ and $2^{3} \mathrm{~S} \rightarrow$ $E^{3} \mathrm{P}$ transitions are presented for the photoelectron energy range $0.15 \leqslant$ $k_{1}^{2} \leqslant 2.4$ in Tables $I$ and $I I$, respectively. Because the evaluations of the two alternative forms of the transition matrix elements are known to depend most strongly on different regions of configuration space, the fact that in most cases the length and velocity results differ by only 
a few percent may be taken as an indication of their reliability. The present results are in good agmeement with the oscillator strengths calculated by Norcross ${ }^{12}$ in the near threshold region, but at the higher energies there are differences of more than 10 percent. Since the same final state wave functions have been employed in both calculations, the descrepancies must be attributed to inaccuracies in the bound state wave functions used by Norcross. 12

The S- and D- wave partial oscillator strengths $\mathrm{df}_{\mathrm{S}} / \mathrm{dE}$ and $\mathrm{df}_{\mathrm{D}} / \mathrm{dE}$ for photoionization from unpolarized $2^{1} \mathrm{P}$ and $2^{3} \mathrm{P}$ states of helium are tabulated for the energy range $0.15 \leqslant k_{1}^{2} \leqslant 2.4$ in Tables III and IV, respectively. The total cross sectlons are obtained by adding the two partial-wave contributions. As anticipated the D partial-wave contribution is dominant near the threshold in both cases, and for the $2^{3} \mathrm{P}$ state the dominance of the ${ }^{3} \mathrm{D}$ - wave partial cross section extends over the entire energy range considered. The increased importance of the ${ }_{2}^{1} \mathrm{P} \rightarrow \mathrm{E}^{1} \mathrm{~S}$ transition at higher energies is attributable mainly to the eccurrence of a zero minimum in the ${ }^{1}$ cross section. The agreement between the length and velocity results is very good except at the zero ininimum.

The total photoionization cross sections for the $2^{1} \mathrm{~S}$ and $2^{3} \mathrm{~S}$ states and for unpolarized $2^{1} \mathrm{P}$ and $2^{3} \mathrm{P}$ states of hellum are shown in Figure 1 for photoelectron energies in the nonresonant region below the $n=2$ threshold. The cross sections for photoionization from the P-states, while decreasing more rapidly away from the first tonization threshold, 
are predicted to be comparable to the sm state cross sections in the near-threshold region, Unfortunately the present numerical procedures do not allow accurate results to be obtained for $k_{1}{ }^{2}<0.15$ as has been achieved in a previous calculation for the $1^{1} S$ and $2^{1} S$ states ${ }^{6}$. However, the cross sections exhibit an approximately linear dependence near the thresholds when plotted as functions of the photon wavelength. An accus. rate extrapolation procedure should therefore be possible.

The resonant features associated with the lowest ${ }^{1} \mathrm{~S},{ }_{\mathrm{P}},{ }_{\mathrm{D}}$, and ${ }_{\mathrm{P}}$ helium autoionizing states are clearly visible in the semi-logarithmic cross section plots presented in Figures 2 and 3. Each partial wave contribution was fitted to the Fano line shape formula ${ }^{23}$

$$
\frac{\mathrm{df}_{\mathrm{L}_{T}}}{\mathrm{dE}}=\left(\frac{\mathrm{df}_{\mathrm{L}_{T}}}{\mathrm{dE}}\right)_{0} \frac{\left(\epsilon+\mathrm{q}_{\mathrm{L}_{T}}\right)^{2}}{1+\epsilon^{2}}
$$

where $\epsilon$ is defined in terms of the resonance position $E_{r}$ and the width $\Gamma_{\mathrm{r}}$ according to the relation

$$
\epsilon=\frac{2\left(\mathrm{E}-\mathrm{E}_{\mathrm{r}}\right)}{\Gamma_{\mathrm{r}}}
$$

The nonresonant background oscillator strength $\left(\mathrm{df}_{\mathrm{L}_{\mathrm{T}}} / \mathrm{dE}\right)_{\mathrm{o}}$ was allowed to vary linearly with $\epsilon$ through the resonance. The line profile parameter $\mathrm{q}_{L_{T}}$ and the background osclilator strength (evaluated at $\epsilon=0$ ) which were obtained for each resonance are given in Table V.

The most important result of the analysis is that the resonant shapes considered are found to be characterized by large values of the line 
profile parameter $\mathrm{q}_{L_{T}}$, partlcularly for the last three transtition in Table $v$. A slgniflcant enhancement of the photoionization cross sections for these three transitions might have been anticipated since they fnvolye singlemelectron excttations. From the result that the value of $\mathrm{q}_{L_{T}}$ is large it follows that the resonant proftle described by equation (12) is more nearly lerentzian and that the $\Delta \mathrm{E}=\Gamma_{\mathrm{r}} / \mathrm{q}_{\mathrm{L}_{\mathrm{T}}}$ displacement of the peak from the resonance postion $E_{r}$ is negligible.

The zero minimum in the ${ }^{A} D$ - wave partial phototonization cross section appears to be the dip associated with the $\left(2 p^{2}\right)^{1}$ D resonance which is predicted by the analytic form (12) to occur at $\epsilon=-\mathrm{q}_{\mathrm{L}_{\mathrm{T}}}$. The occurrence of the suppression of the D- wave photolonization continuum several times the width below the resonance position is therefore attributable to the large positive value of the line profile parameter: In the case of the helium metastable states, Table $V$ indicates some rather large descrepances between the line profile parameters and background esclilator strengths obtained from the present calculations and the values repurted by Norcross. ${ }^{12}$ This descrepanctes may be accounted for by the assumption that $\mathrm{q}_{L_{T}}$ is energy-independent which, as has been peinted out by Norcross ${ }^{12}$, may not be valid for large values of $\mathrm{q}_{\mathrm{L}_{\mathrm{T}}}$. For a polarized $\mathrm{L}_{\mathrm{O}^{-}}$state of the helium atom, the total photoionization cross section may st 111 be expressed as an incoherent superposition of individual $\mathrm{L}_{\mathrm{T}^{-}}$wave contributions given by equation (10) provided the factor $1 / 3\left(2 L_{o}+1\right)$ in the definition of the oscillator strength (11) is replaced by

$$
(-1)^{1+L_{0}+L_{T}} \sum_{K} \rho_{o}(K, o) \rho_{r}(K, o)\left\{\begin{array}{ccc}
L_{o} & L_{o} & K \\
1 & 1 & L_{T}
\end{array}\right\}
$$


As an fllustration of the effect of the atomic polarization for the case of unpolapized 1fght, the photoionization cross sections for unpolarized and for aligned $\left(M L_{0}=122^{1} p\right.$ and $2^{3} p$ states of helium are compared in Table VI.

In the case of phototontzation from aligned helium $P$ - states by right circularly (IInearly) polarized photons, with the atomic quantization axis chosen along the photon propogation (polarization) direction, only the transition to the $D$ - wave continuum w111 occur. The photoionization cross sections may then be obtained from equation (10) provided the Dwave contributions given in Tables III and IV are first multiplied by 1.8 and 0.9 for the circular and linear polarizations respectively. It is apparent that a measurement of the photolonization cross sections for unpolarized and for allgned P-istates of helfum with circularly or Iinearly polarized light would lead to separate determinations of the Individual S- and D- partial wave contributions. 
$V$ The Asymmetry; Parameters

In the experimental inyestigation of photolonization from the Pm states of helium by photoelectron spectroscopy techniques whtch detect only the electrons ejected In a given direction, a knowledge of the asymetry parameters would be required in order to relate the observed electron intensity to the total cross sections. The calctlated values of the asymetry parameter $\beta$ for unpolarized $2^{1} \mathrm{P}$ and $2^{3} \mathrm{P}$ states of helium are presented in Table VII for the photoelectron energy range $0.15 \leqslant k_{1}{ }^{2} \leqslant$ 2.4 and are shown over a more extended energy range in Figure 4 . The asymmetry parameters $\beta_{2}$ and $\beta_{4}$ for photoionization of aligned $2^{1} \mathrm{P}$ and $2^{3} \mathrm{P}$ states of helium by unpolarized light are shown in Figure 5.

For ionization of aligned P- states by right circularly polarized photons the energy-independent values of the asymetry parameters are found to be $\beta_{2}=-1.429$ and $\beta_{4}=0.4286$, while in the case of linearly polarized light the values are $\beta_{2}=0.7143$ and $\beta_{4}=-1.714$.

The most striking features occur in the $2^{1} \mathrm{p}$ case, where the lowest $1_{S}$ and ${ }^{1} D$ helium resonances produce conspicupus eaergy variations in the asymmetry parameters. For an understanding of the behavior of the $2^{1} P$ asymmetry parameter $\beta$ illustrated in Figure 4, it may be noted that the hypothetical values of $\beta$ associated with pure $S$ and $D$ transitions are 0 and 1 , respectively. 
VI Conclustons

The phototonization cross sections presented in this paper should be accurate to within 10 percent or betten. The introduction of Hy1leraas terms into the final state continum wave functions would lead to greater precision and a more balianced calculation.

Taking into consfderation the available experimental techniques, it appears desirable to attempt a measurement of the $2^{1} \mathrm{P}$ helium photom ionization cross section by excitation of a helium gas with $2^{1} P-1^{1} S$ resonance radiation from a helium discharged and subsequent ionization using a tunable laser. 
Acknowledgements

I whish to acknowledge Brofessor R.G, Burke's important contrtbution to the present calculations, which eowld not have been performed without the use of his collision code.

I would also like to thank Mr, R, Baxter, Mr. J. Bredekamp, and Mr. E. Sullivan for thetr generous assistance in setting up the computer programs at the Goddard Space F1ight Center. 
Table 1. The oscillator strength for the $2^{1}$ s state of helium. The length and velocity results are denoted by $L$ and $V$, respectively. The numbers in parenthesis are the powers of ten by which the entries are to be multiplied.

\begin{tabular}{lll}
\hline $\mathrm{k}_{1}{ }^{2}(\mathrm{RY})$ & $\left(\frac{\mathrm{df}}{\mathrm{de}}\right)_{\mathrm{L}}$ & $\left(\frac{\mathrm{df}}{\mathrm{dE}}\right)_{\mathrm{V}}$ \\
\hline 0.15 & 0.5545 & 0.5903 \\
0.20 & 0.4736 & 0.4805 \\
0.25 & 0.4008 & 0.4022 \\
0.30 & 0.3431 & 0.3402 \\
0.40 & 0.2564 & 0.2519 \\
0.60 & 0.1528 & 0.1503 \\
0.80 & $0.9842(-1)$ & $0.9713(-1)$ \\
1.00 & $0.6737(-1)$ & $0.6635(-1)$ \\
1.20 & $0.4812(-1)$ & $0.4696(-1)$ \\
1.40 & $0.3526(-1)$ & $0.3398(-1)$ \\
1.60 & $0.2611(-1)$ & $0.2480(-1)$ \\
1.80 & $0.1922(-1)$ & $0.1797(-1)$ \\
2.00 & $0.1364(-1)$ & $0.1254(-1)$ \\
2.20 & $0.8589(-2)$ & $0.7695(-2)$ \\
2.40 & $0.2869(-2)$ & $0.2372(-2)$
\end{tabular}


Table II. The oscillator strength for the $2^{3} \mathrm{~S}$ state of helium. The length and velocity results are denoted by $\mathrm{L}$ and $\mathrm{V}$, respectively. The numbers in parenthesis are the powers of ten by which the entries are to be multiplied.

\begin{tabular}{lll}
\hline $\mathrm{k}_{1}{ }^{2}(\mathrm{RY})$ & $\left(\frac{\mathrm{df}}{\mathrm{dE}}\right)_{\mathrm{L}}$ & $\left(\frac{\mathrm{df}}{\mathrm{dE}}\right)_{\mathrm{V}}$ \\
\hline 0.15 & 0.4385 & 0.4498 \\
0.20 & 0.3914 & 0.4111 \\
0.25 & 0.3466 & 0.3642 \\
0.30 & 0.3074 & 0.3235 \\
0.40 & 0.2442 & 0.2567 \\
0.60 & 0.1625 & 0.1712 \\
0.80 & 0.1148 & 0.1211 \\
1.00 & $0.8491(-1)$ & $0.8960(-1)$ \\
1.20 & $0.6527(-1)$ & $0.6882(-1)$ \\
1.40 & $0.5183(-1)$ & $0.5460(-1)$ \\
1.60 & $0.4244(-1)$ & $0.4464(-1)$ \\
1.80 & $0.3588(-1)$ & $0.3766(-1)$ \\
2.00 & $0.3164(-1)$ & $0.3311(-1)$ \\
2.20 & $0.3038(-1)$ & $0.3162(-1)$ \\
2.40 & $0.4648(-1)$ & $0.4768(-1)$
\end{tabular}


Table III. The S- and D- wave partial oscillator strengths for the $2^{1} \mathrm{P}$ state of helium. The length and velocity results are denoted by $\mathrm{L}$ and $\mathrm{V}$, respectively. The numbers in parentheses are the powers of ten by which the entries are to be multiplied.

\begin{tabular}{|c|c|c|c|c|}
\hline $\mathrm{k}_{1}^{2}(\mathrm{RY})$ & $\left(\frac{d f}{d E}\right)_{L}$ & $\left(\frac{\mathrm{df}}{\mathrm{dE}} \mathrm{s}\right)_{V}$ & $\left(\frac{d f_{D}}{d E}\right)_{L}$ & $\left(\frac{\mathrm{df}}{\mathrm{dE}}\right)_{\mathrm{V}}$ \\
\hline 0.15 & $0.3467(-1)$ & $0.3720(-1)$ & 0.3350 & 0.4201 \\
\hline 0.20 & $0.2621(-1)$ & $0.2634(-1)$ & 0.2196 & 0.2211 \\
\hline 0.25 & $0.2033(-1)$ & $0.2030(-1)$ & 0.1540 & 0.1527 \\
\hline 0.30 & $0.1626(-1)$ & $0.1598(-1)$ & 0.1107 & 0.1081 \\
\hline 0.40 & $0.1091(-1)$ & $0.1043(-1)$ & $0.6027(-1)$ & $0.5733(-1)$ \\
\hline 0.60 & $0.5625(-2)$ & $0.5178(-2)$ & $0.2060(-1)$ & $0.1913(-1)$ \\
\hline 0.80 & $0.3288(-2)$ & $0.2988 \quad(-2)$ & $0.7940(-2)$ & $0.7222(-2)$ \\
\hline 1.00 & $0.2114(-2)$ & $0.1935(-2)$ & $0.3201(-2)$ & $0.2810(-2)$ \\
\hline 1.20 & $0.1486(-2)$ & $0.1380(-2)$ & $0.1247(-2)$ & $0.1010(-2)$ \\
\hline 1.40 & $0.1134(-2)$ & $0.1075(-2)$ & $0.3970(-3)$ & $0.2663(-3)$ \\
\hline 1.60 & $0.9465(-3)$ & $0.9154(-3)$ & $0.5795(-4)$ & $0.1507(-4)$ \\
\hline 1.80 & $0.8787(-3)$ & $0.8652(-3)$ & $0.1306(-4)$ & $0.5505 \quad(-4)$ \\
\hline 2.00 & $0.9578(-3)$ & $0.9540(-3)$ & $0.2489(-3)$ & $0.3841(-3)$ \\
\hline 2.20 & $0.1463(-2)$ & $0.1466(-2)$ & $0.1091(-2)$ & $0.1347(-2)$ \\
\hline 2.40 & $0.1436(-1)$ & $0.1433(-1)$ & $0.5340(-2)$ & $0.5815(-2)$ \\
\hline
\end{tabular}


Table IV. The S- and D-wave partial oscillator strengths for the $2{ }^{3} \mathrm{P}$ state of helium. The length and velocity results are denoted by $L$ and $V$, respectively. The numbers in parentheses are the powers of ten by which the entries are to be multiplied.

\begin{tabular}{|c|c|c|c|c|c|}
\hline $\mathrm{k}_{1}^{2}(\mathrm{RY})$ & $\left(\frac{\mathrm{df} s}{\mathrm{dE}}\right)_{L}$ & $\left(\frac{\mathrm{df}}{\mathrm{dE}}\right)_{V}$ & $\left(\frac{d f_{D}}{d E}\right)_{L}$ & $\left(\frac{d f}{d E}\right)_{V}$ & \\
\hline 0.15 & $0.4146(-1)$ & $0.4825(-1)$ & 0.4484 & & \\
\hline 0.20 & $0.3280(-1)$ & $0.3801(-1)$ & 0.3617 & 0.3715 & \\
\hline 0.25 & $0.2546(-1)$ & $0.2780(-1)$ & 0.2691 & 0.2664 & \\
\hline 0.30 & $0.2046(-1)$ & $0.2235(-1)$ & 0.2058 & 0.2013 & \\
\hline 0.40 & $0.1404(-1)$ & $0.1485(-1)$ & 0.1272 & 0.1223 & \\
\hline 0.60 & $0.7237(-2)$ & $0.7489(-2)$ & $0.5658(-1)$ & 0.5367 & $(-1)$ \\
\hline 0.80 & $0.4149(-2)$ & $0.4253(-2)$ & $0.2917(-1)$ & 0.2778 & $(-1)$ \\
\hline 1.00 & $0.2512(-2)$ & $0.2612(-2)$ & $0.1678(-1)$ & 0.1608 & $(-1)$ \\
\hline 1.20 & $0.1606(-2)$ & $0.1701(-2)$ & $0.1052(-1)$ & 0.1010 & $(-1)$ \\
\hline 1.40 & $0.1073(-2)$ & $0.1156(-2)$ & $0.7043(-2)$ & 0.6748 & $(-2)$ \\
\hline 1.60 & $0.7290(-3)$ & $0.8104(-3)$ & $0.4975(-2)$ & 0.4734 & $(-2)$ \\
\hline 1.80 & $0.5127(-3)$ & $0.5819(-3)$ & $0.3669(-2)$ & 0.3459 & $(-2)$ \\
\hline 2.00 & $0.3666(-3)$ & $0.4242(-3)$ & $0.2809(-2)$ & 0.2617 & $(-2)$ \\
\hline 2.20 & $0.2634(-3)$ & $0.3110(-3)$ & $0.2222(-2)$ & 0.2047 & $(-2)$ \\
\hline 2.40 & $0.1869(-3)$ & $0.2255(-3)$ & $0.1819(-2)$ & 0.1659 & $(-2)$ \\
\hline
\end{tabular}


Table V. The line profile parameter $\mathrm{q}_{\mathrm{LT}}$ and the background oscillator strength (df $\left.\mathrm{L}_{\mathrm{f}} / \mathrm{dE}\right)_{\mathrm{O}}$ obtained for the lowest-lying ${ }_{\mathrm{S}},{ }^{3} \mathrm{P}$, $1_{P}$, and ${ }^{1} \mathrm{D}$ helium resonances using the length matrix elements. The numbers in parenthesis are the powers of ten by which the entries are to be multiplied

\begin{tabular}{|c|c|c|c|c|}
\hline Transition & $\mathrm{E}_{r}(\mathrm{eV})^{\mathrm{a}}$ & $\Gamma_{r}(\mathrm{eV})^{\mathrm{a}}$ & $\mathrm{q}_{\mathbf{L}_{\mathbf{T}}}$ & $\left(\frac{\mathrm{df}}{\mathrm{dE}} \mathrm{L}_{\mathrm{T}}\right)_{\mathrm{O}}$ \\
\hline $2^{1} p \rightarrow\left(2 s^{2}\right)^{1} s$ & $57.865^{\dagger}$ & $1.41(-1)$ & -29.30 & $9.487(-4)$ \\
\hline $2^{3} S \rightarrow(2 s 2 p)^{3} p$ & 58.360 & $1.06(-2)$ & $\begin{array}{l}-78.37 \\
-106.6^{b}\end{array}$ & $\begin{array}{l}3.711(-2) \\
2.17(-2)^{b}\end{array}$ \\
\hline $2^{1} \mathrm{q} \rightarrow\left(2 \mathrm{p}^{2}\right)^{1} \mathrm{D}$ & 60.025 & $7.32(-2)$ & 172.4 & $7.792(-4)$ \\
\hline $2^{1} s+(2 s 2 p)^{1} p$ & 60.269 & $4.38(-2)$ & $\begin{array}{l}44.68 \\
64.73_{b}\end{array}$ & $\begin{array}{l}1.431(-2) \\
1.29(-2)^{b}\end{array}$ \\
\hline
\end{tabular}

$+\mathbf{E}_{\mathbf{r}}$ is measured with respect to the helium ground state.

a Reference 11 .

b Reference 12 . 
Table VI. The photoionization cross sections (in units of $10^{-18} \mathrm{~cm}^{2}$ ) for unpolarized and polarized $\left(\mathrm{ML}_{\mathrm{O}}=1\right)$ P-states of helium. evaluated using the length matrix elements. The numbers in parentheses are the powers of ten by which the entries are to be multiplied.

\begin{tabular}{|c|c|c|c|c|}
\hline \multirow[b]{2}{*}{$\mathrm{k}_{1}{ }^{2}(\mathrm{RY})$} & \multicolumn{2}{|c|}{ unpolarized He atoms } & \multicolumn{2}{|c|}{ polarized He atoms $\dagger$} \\
\hline & $2^{1} \mathrm{P}$ & $2^{3} \cdot p$ & $2^{1} P$ & $2^{3} \mathrm{P}$ \\
\hline 0.15 & 2.983 & 3.952 & 3.258 & 4.300 \\
\hline 0.20 & 1.983 & 3.183 & 2.178 & 3.461 \\
\hline 0.25 & 1.406 & 2.376 & 1.550 & 2.587 \\
\hline 0.30 & 1.024 & 1.825 & 1.134 & 1.991 \\
\hline 0.40 & $5.743(-1)$ & 1.139 & $6.426(-1)$ & 1.247 \\
\hline 0.60 & $2.116(-1)$ & $5.148(-1)$ & $2.426(-1)$ & $5.668(-1)$ \\
\hline 0.80 & $9.057(-2)$ & $2.688(-1)$ & $1.070(-1)$ & $2.973(-1)$ \\
\hline 1.00 & $4.288(-2)$ & $1.556(-1)$ & $5.269(-2)$ & $1.725(-1)$ \\
\hline 1.20 & $2.204(-2)$ & $9.778(-2)$ & $2.854(-2)$ & $1.085(-1)$ \\
\hline 1.40 & $1.235(-2)$ & $6.547(-2)$ & $1.708(-2)$ & $7.264(-2)$ \\
\hline 1.60 & $8.103(-3)$ & $4.602(-2)$ & $1.194(-2)$ & $5.096(-2)$ \\
\hline 1.80 & $7.194(-3)$ & $3.374(-2)$ & $1.074(-2)$ & $3.729(-2)$ \\
\hline 2.00 & $9.735(-3)$ & $2.562(-2)$ & $1.374(-2)$ & $2.823(-2)$ \\
\hline 2.20 & $2.061(-2)$ & $2.005(-2)$ & $2.695(-2)$ & $2.201(-2)$ \\
\hline 2.40 & $1.589(-1)$ & $1.618(-2)$ & $2.190(-1)$ & $1.767(-2)$ \\
\hline
\end{tabular}

t unpolarized photons 
Table VII. The photoelectron angular distribution asymetry parameter $\beta$ for unpolarized $2^{1} \mathrm{P}$ and $2^{3} \mathrm{P}$ states of helium. The length and velocity results are denoted by $L$ and $V$, respectively. The numbers in parentheses are the powers of ten by which the entries are to be multiplied.

\begin{tabular}{|c|c|c|c|c|}
\hline $\mathrm{k}_{1}{ }^{2}(\mathrm{RY})$ & ${ }^{B} \mathrm{~L}$ & ${ }^{\beta} \mathrm{V}$ & $\beta_{L}$ & ${ }^{B} V$ \\
\hline 0.15 & 1.566 & 1.538 & 1.696 & \\
\hline 0.20 & 1.521 & 1.520 & 1.673 & 1.703 \\
\hline 0.25 & 1.468 & 1.469 & 1.657 & 1.679 \\
\hline 0.30 & 1.416 & 1.416 & 1.640 & 1.664 \\
\hline 0.40 & 1.310 & 1.311 & 1.600 & 1.619 \\
\hline 0.60 & 1.095 & 1.096 & 1.504 & 1.515 \\
\hline 0.80 & $8.699(-1)$ & $8.701(-1)$ & 1.404 & 1.410 \\
\hline 1.00 & $6.269(-1)$ & $6.170(-1)$ & 1.308 & 1.311 \\
\hline 1.20 & $3.594(-1)$ & $3.266(-1)$ & 1.220 & 1.221 \\
\hline 1.40 & $8.690(-2)$ & $4.163(-2)$ & 1.142 & 1.140 \\
\hline 1.60 & $-7.228(-2)$ & $-5.417(-2)$ & 1.074 & 1.068 \\
\hline 1.80 & $9.796(-2)$ & $2.243(-1)$ & 1.016 & 1.005 \\
\hline 2.00 & $5.322(-1)$ & $6.514(-1)$ & $9.675(-1)$ & $9.504(-1)$ \\
\hline 2.20 & $8.659(-1)$ & $9.220(-1)$ & $9.283(-1)$ & $9.058(-1)$ \\
\hline 2.40 & $6.019(-1)$ & $6.260(-1)$ & $9.000(-1)$ & $8.727(-1)$ \\
\hline
\end{tabular}




\section{References}

1. H. R. Griem, Plasma Spectroscopy (McGraw-Hill, New York. 1964).

2. V. P. Myerscough and G. Peach, Case Studies In Atomic Collision Physics II (North-Holland, Amsterdam, 1972).

3. J. Bakos, A. Kiss; L. Szabo, and M. Tendler, Phys. Lett. 39A, 317 (1972).

4. R. F. Stebbings, F. B. Dunning, F. K. Tittel, and R. D. Runde1, Phys. Rev. Lett. 30, 815 (1973).

5. V. L. Jacobs, Phys. Rev. $\underline{A} 3,289$ (1971).

6. V. L. Jacobs, Phys. Rev. A4, 939 (1972).

7. V. L. Jacobs and P. G. Burke, J. Phys. B Atom. Molec. Phys, 5, L67 (1972).

8. V. L. Jacobs and P. G. Burke, J. Phys. B Atom. Molec. Phys, $\underline{5}$, 2272 (1972).

9. H. A. Hyman, V. L. Jacobs, and P. G. Burke, J. Phys. B Atom. Molec. Phys. $\underline{5}, 2282$ (1972).

10. M. O. Krause and F. Wuilleumier, J. Phys. B Atom. Molec. Phys. $\underline{5}$, L143 (1972).

11. P. G. Burke and D. D. McVicar, Proc. Phys. Soc., London $\underline{80}, 616$ (1962).

12. D. W. Norcross, J. Phys. B 4 , 652 (1971).

13. L. Goldberg, Astrophys. J., 90, 414 (1939).

14. A. Burgess and M. J. Seaton, Mon. Not. Roy. Astr. Soc. $\underline{120}, 121$ (1960). 
15.a. Anderson and H. R. Griem, Ionization Phenomena in Gases, $\underline{3}$, 293 (North-Holland, Amsterdam, 1963).

16. H.A. Bethe and E. E. Salpeter, Quantum Mechanics of One- and TwoElectron Atoms (Academic, New York, 1957).

17. J. Cooper and R. N. Zare, J. Chem. Phys. $\underline{48}, 942$ (1968).

18. U. Fano and D. D111, Phys. Rev. A6, 185 (1972).

19. V. L. Jacobs, J. Phys. B. $\underline{5}, 2257$ (1972).

20. U. Fano and G. Racah, Irreducible Tensorial Sets (Academic, New York, 1959).

21. Y. Accad, C. L. Pekeris, and B. Schiff, Phys. Rev. A4, 516 (1971).

22. P. G. Burke, The Physics of Electronic and Atomic Collisions (J.I.L.A., Boulder, 1968).

23. U. Fano, Phys. Rev. 124, 1866 (1961). 


\section{Figure Captions}

Fig. 1. The photoionization cross sections for the $2^{1} S$ and $2^{3} S$ states and for unpolarized $2^{\mathrm{P}} \mathrm{P}$ and $2^{3} \mathrm{P}$ states of helium in the nonresonant region, evaluated using the length matrix elements.

Fig. 2. Resonant features in the photolonization cross sections for the $2^{1} S$ state and for an unpolarized $2^{1} \mathrm{P}$ state of helium.

Fig. 3. The $(2 \mathrm{~s} 2 \mathrm{p})^{3} \mathrm{P}$ resonance in the $2^{3} \mathrm{~S}$ helium photoionization cross section and the photoionization cross section for an unpolarized $2^{3} \mathrm{P}$ state of hellum.

Fig. 4. The asymmetry parameter $\beta$ for photoionization of unpolarized ${ }_{2}^{1} \mathrm{P}$ and $2^{3} \mathrm{P}$ states of helium, evaluated using the length matrix elements.

Fig. 5. The asymmetry parameters $\beta_{2}$ and $\beta_{4}$ for photolonization of aligned $2^{1} \mathrm{P}$ and $2^{3} \mathrm{P}$ states of helium by unpolarized light, evaluated using the length matrix elements. 


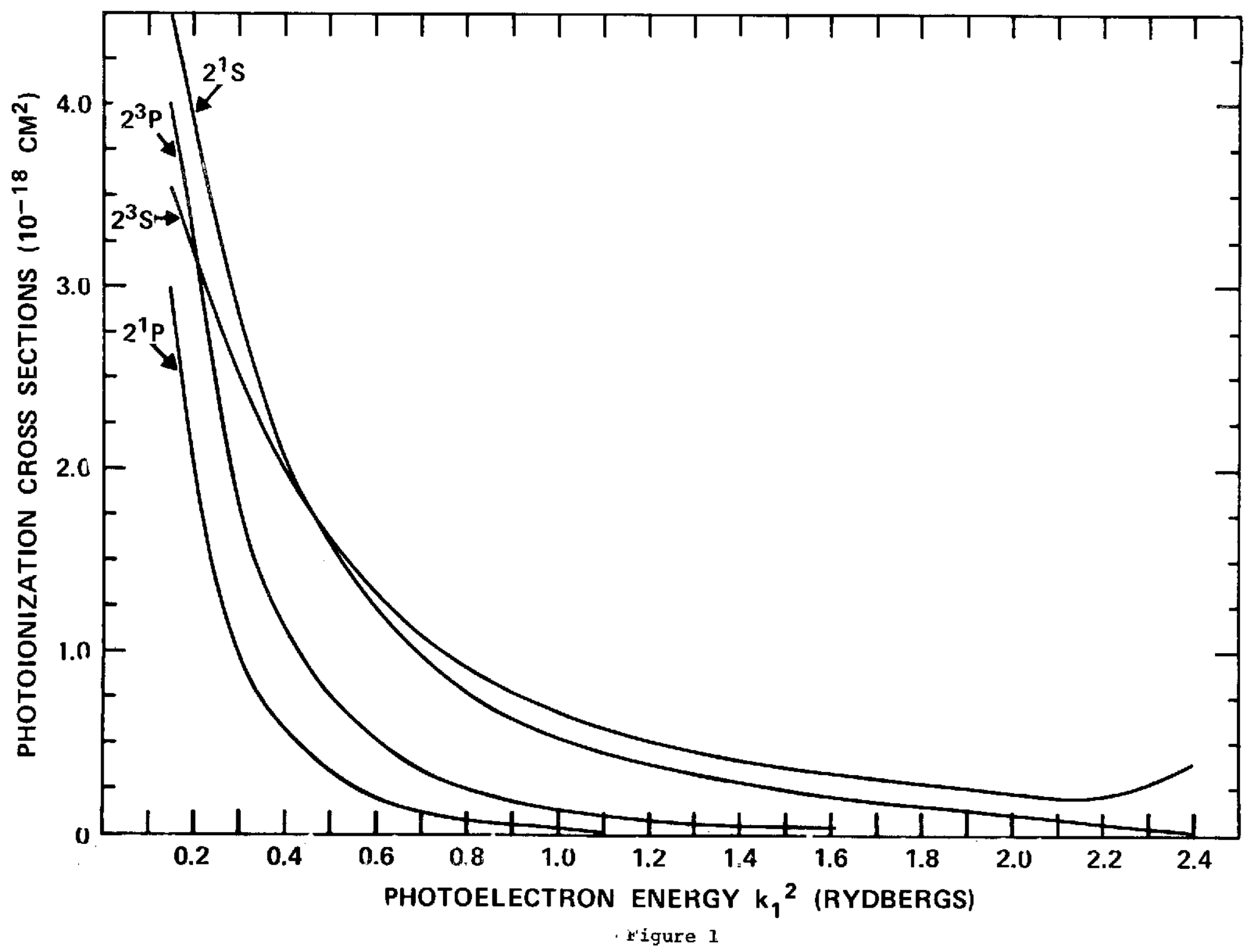

$\underline{w}$ 


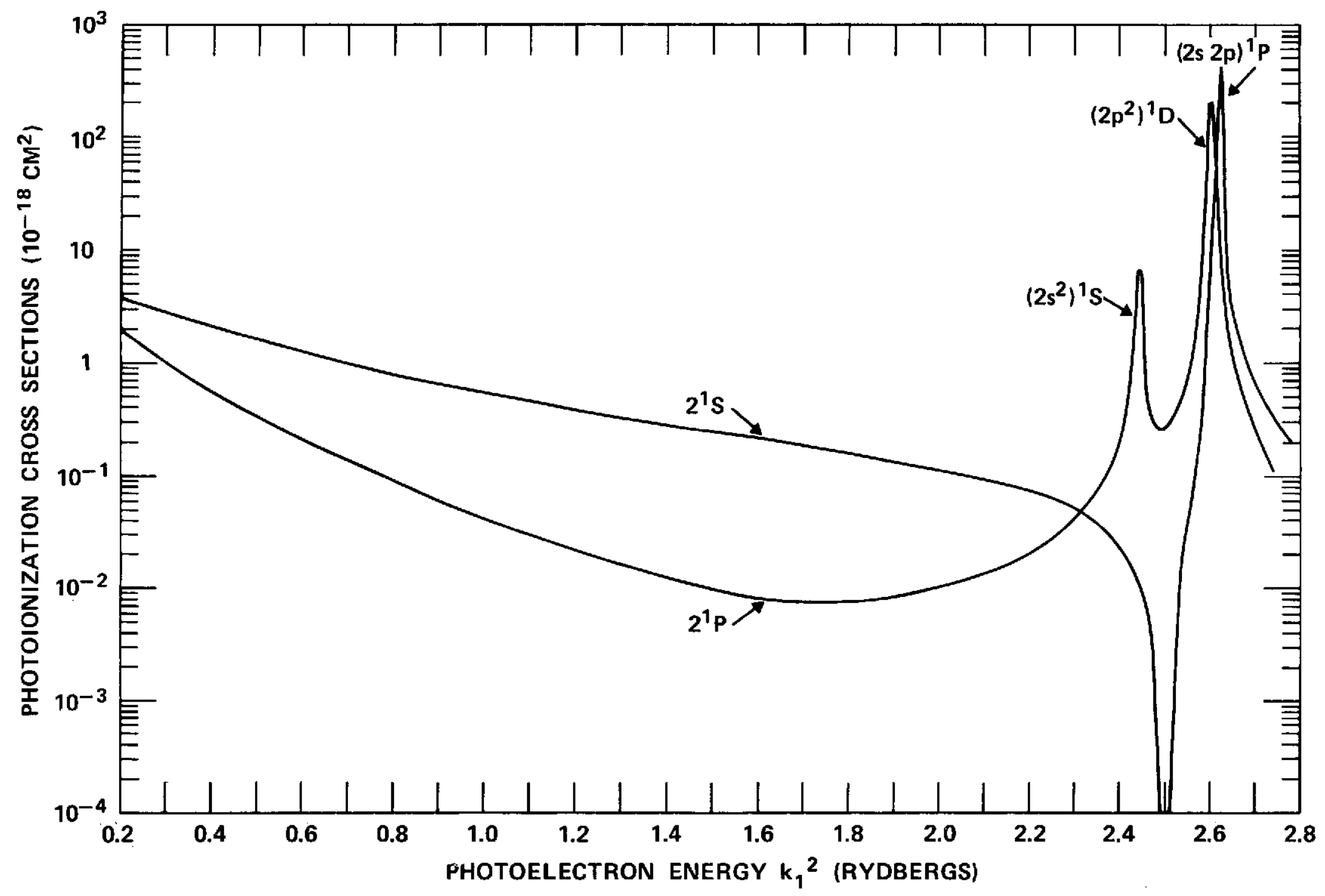

Figure 2 


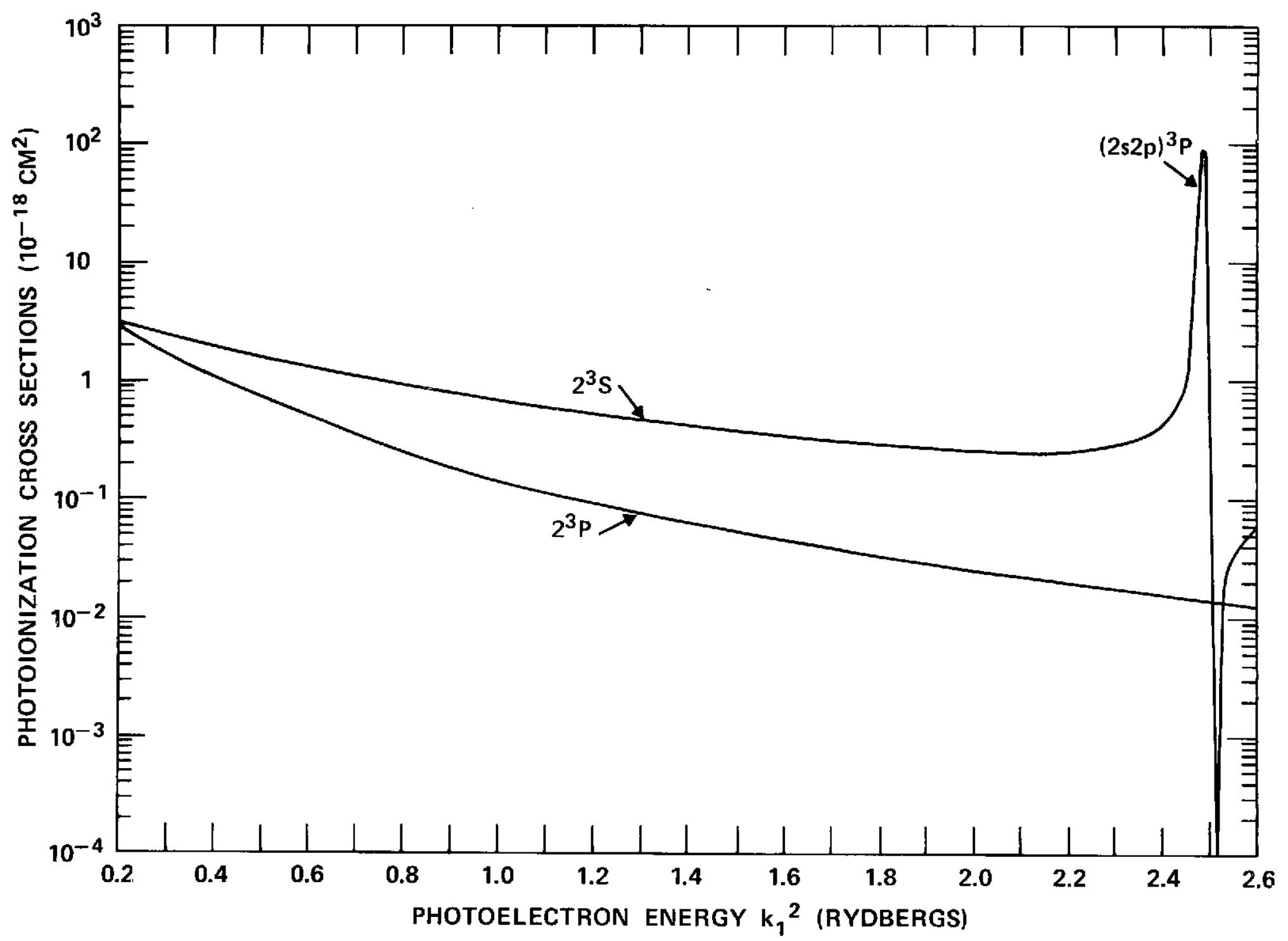

Figure 3 


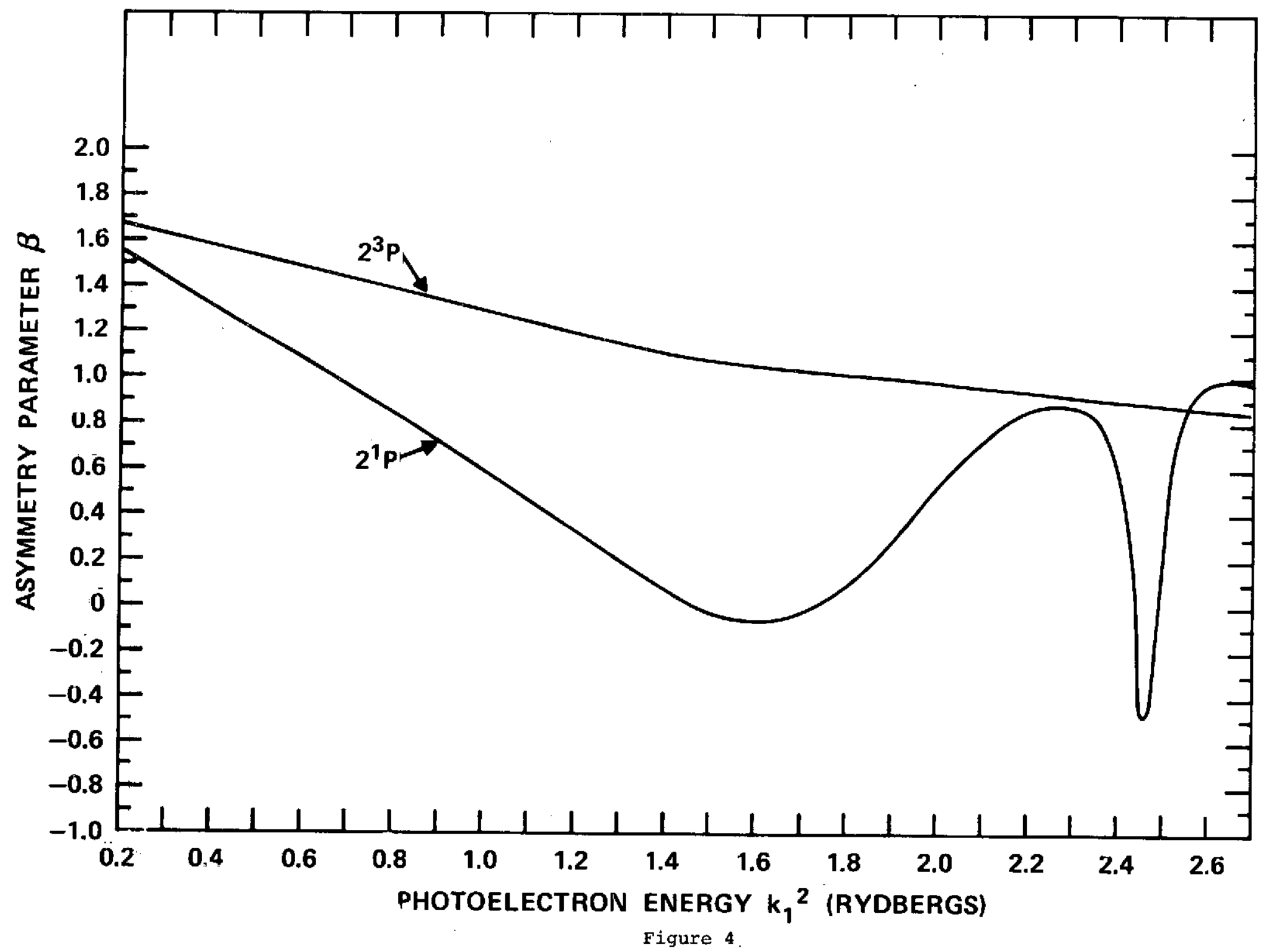




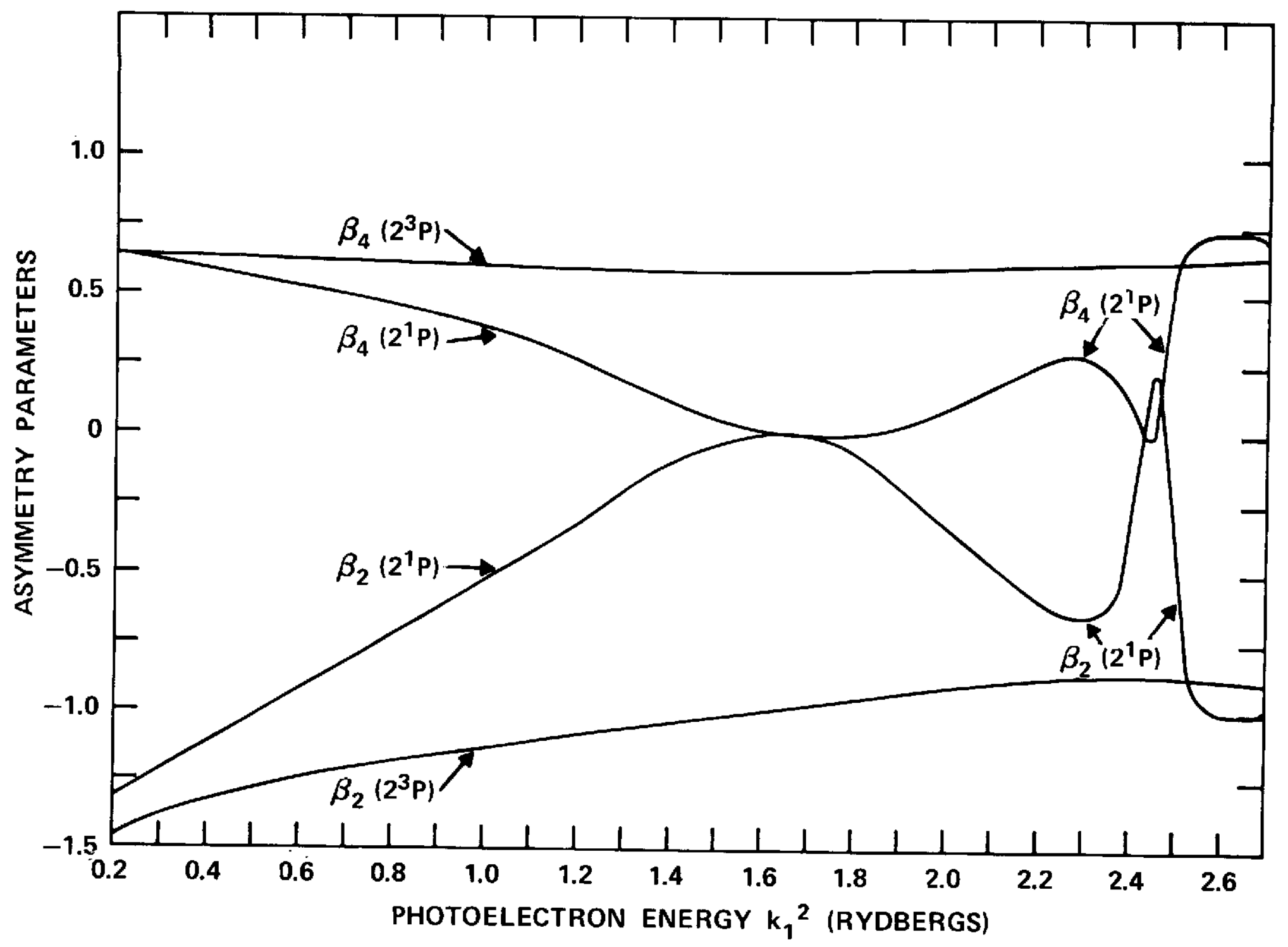

Figure 5 